\title{
Vortex and source particles for fluid motion estimation
}

\author{
Anne Cuzol and Etienne Mémin \\ IRISA, Université de Rennes 1, \\ Campus de Beaulieu, \\ 35042 Rennes Cedex, France \\ \{acuzol, memin\}@irisa.fr
}

\begin{abstract}
In this paper we propose a new motion estimator for image sequences depicting fluid flows. The proposed estimator is based on the Helmholtz decomposition of vector fields. This decomposition consists in representing the velocity field as a sum of a divergence free component and a curl free component. In order to provide a low dimensional solution, both components are approximated using a discretization of the vorticity and divergence maps through regularized Dirac measure. The resulting so called irrotational and solenoidal fields consist then in linear combinations of basis functions obtained through a convolution product of the Green kernel gradient and the vorticity map or the divergence map respectively. The coefficient values and the basis function parameters are obtained as the minimizer of a functional relying on an integrated version of mass conservation principle of fluid mechanics. Results are provided on real world sequences.
\end{abstract}

\section{Introduction}

The observation, understanding and control of complex fluid flows is a major scientific issue. For instance, in environmental sciences such as oceanography, meteorology and climatology, the monitoring or the forecasting of the atmosphere or the ocean is becoming more and more crucial for our everyday life. Due to their very complex nature and also to unknown or inaccurate border conditions, we have a lack of complete physical understanding of these flows. Accurate and dense measurements can hardly be recovered by probes or by numerical evaluation of current physical models. Imaging sensors are very attractive in this context as they provide multi-modal data at high spatio-temporal resolution.

The analysis of dynamic structures and the estimation of velocities for fluid image sequences gave rise to a great attention from the computer vision community since several years $[6,7,10,12,14,15]$. These works concern application domains such as experimental visualization in fluid mechanics, environmental sciences (oceanography, meteorology, ...), or medical imagery.

Recently, several dedicated approaches have been proposed for fluid flow velocity estimation $[4,9]$. Unlike most of the motion estimator based on the brightness consistency assumption and a first order smoothness function, these techniques rely on a data-model derived from the continuity equation of fluid mechanics and second order div-curl regularizers. In the same way as a first order regularizer (eventually associated to a robust cost function) favors piecewise translational motion fields in penalizing high gradients of the solution, second order div-curl penalizers encourage solutions with blobs of piecewise constant divergence and curl. These methods are conceptually 
much more satisfying as they comply with the brightness variations and the motions observed in fluid image sequences. Nevertheless, these method have to face much greater numerical complexity. Besides, these estimators are dense estimator and the solutions associated belong therefore to spaces of great dimension. It is desirable for some applications to provide low dimensional solutions. This is the purpose of this paper.

We propose here a technique to estimate low dimensional motion field from image sequences depicting a fluid flow. This method relies on the Helmholtz decomposition of a motion field which consists to decouple the vector into a divergence free component and a curl free component. The method we devise is based on a discrete representation of the curl (also called vorticity) and divergence map. This discretization enables to define implicitly adapted regularizers for fluid motion estimation problems.

\section{Definitions and properties of vector fields}

In this section, we present first known analytic results on planar vector fields. We shall rely on them to develop an original method for fluid motion estimation.

A two-dimensional vector field $\mathbf{w}$ is a $\mathbb{R}^{2}$-valued map defined on a bounded set $\Omega$ of $\mathbb{R}^{2}$. We denote it $\mathbf{w}(\mathbf{x})=(u(\mathbf{x}), v(\mathbf{x}))^{T}$, where $\mathbf{x}=(x, y)$ and $x$ and $y$ are the spatial coordinates. Each component of the vector field will be supposed twice continuously differentiable: $u, v \in C^{2}(\Omega, \mathbb{R})$.

Noting $\boldsymbol{\nabla}=\left(\frac{\partial}{\partial x}, \frac{\partial}{\partial y}\right)$ the operator whose components are the partial derivatives with respect to the coordinates $x$ and $y$, we define the divergence: $\operatorname{div} \mathbf{w}=\frac{\partial u}{\partial x}+\frac{\partial v}{\partial y}=$ $\boldsymbol{\nabla} \cdot \mathbf{w}$ and the scalar vorticity of the vector field: curl $\mathbf{w}=\frac{\partial u}{\partial y}-\frac{\partial v}{\partial x}=\boldsymbol{\nabla} \cdot \mathbf{w}^{\perp}$, where $\mathbf{w}^{\perp}=(-v, u)$ is the orthogonal counterpart of $\mathbf{w}$.

The vorticity accounts for the presence of a rotating motion, while the divergence is related to the presence of sinks or sources in the flow. A vector field whose divergence is null at every point is called solenoidal. Similarly, a field with zero vorticity will be called irrotational. It is well known that for irrotational fields there exists a scalar function $\phi$, called the velocity potential, such that $\boldsymbol{w}=\boldsymbol{\nabla} \phi$. Similarly, for solenoidal fields there exists a scalar function $\psi$ called the stream function such that $\boldsymbol{w}^{\perp}=\nabla \psi$. Any continuous vector field that vanishes at infinity can be decomposed into a sum of an irrotational component with null vorticity and a solenoidal component with null divergence. This is called the Helmholtz Decomposition. When the null border condition can not be imposed, an additional component, named the laminar component, which is both irrotational and solenoidal, has to be included. The decomposition reads then: $\mathbf{w}=\mathbf{w}_{\text {irr }}+\mathbf{w}_{\text {sol }}+\mathbf{w}_{\text {lam }}$. This last component can be approximated using the Horn and Schunck estimator with a strong regularization coefficient [5]. In the sequel we will assumed that the laminar component has been previously computed and that its associated motion has been removed from the image sequence. We will consequently assume a null boarder condition at infinity knowing that the image sequence, $I(\mathbf{x}, t)$, is related to the original image sequence, $I_{o}(\mathbf{x}, t)$, by $I(\mathbf{x}, t)=I_{o}\left(\mathbf{x}+\mathbf{w}_{\text {lam }}(\mathbf{x}, t), t\right)$.

Substituting the two components $\mathbf{w}_{i r r}$ and $\mathbf{w}_{\text {sol }}$ by their expressions in terms of potential functions and considering the divergence and the curl of the motion field enables 
to write the potential function as solution of two Poisson equations:

$$
\Delta \phi=\operatorname{divw}_{i r r} \text { and } \Delta \psi=\operatorname{curlw}_{\text {sol }},
$$

where $\Delta$ denotes the Laplacian operator These solutions may be expressed as convolution products:

$$
\begin{aligned}
\phi & =\int G(\mathbf{x}-\mathbf{u}) \operatorname{div} \mathbf{w}_{i r r}(\mathbf{u}) d \mathbf{u}=G \otimes \operatorname{div} \mathbf{w}_{i r r}, \\
\psi & =\int G(\mathbf{x}-\mathbf{u}) \operatorname{curl} \mathbf{w}_{s o l}(\mathbf{u}) d \mathbf{u}=G \otimes \operatorname{curl} \mathbf{w}_{s o l},
\end{aligned}
$$

where $G$ is the Green's function associated to the two-dimensional Laplacian:

$$
G(\mathbf{x})=\frac{1}{2 \pi} \ln (|\mathbf{x}|) .
$$

As the vector fields $\mathbf{w}_{i r r}$ and $\mathbf{w}_{\text {sol }}$ are respectively the gradient and the orthogonal gradient of the potential functions $\phi$ and $\psi$, equation (2-3) may be rewritten as:

$$
\mathbf{w}_{i r r}=K \otimes \operatorname{div} \mathbf{w}_{i r r} \text { and } \mathbf{w}_{\text {sol }}=K^{\perp} \otimes \operatorname{curl} \mathbf{w}_{\text {sol }},
$$

where $K$ denotes the gradient of the Green kernel. The second equation of (5) is known as the Bio-Savart integral. These two equations state that the solenoidal and the irrotational components (and consequently the whole vector field) may be recovered through a convolution product knowing the divergence and the vorticity of the velocity field.

\section{Vortex particles}

The idea of vortex particles methods $[2,11]$ consists in approximating the vorticity of a field $\mathbf{w}$ by a discrete sum of delta functions located at point vortices $\mathbf{x}_{i}$ :

$$
\operatorname{curl} \mathbf{w}(\mathbf{x}) \approx \sum_{i=0}^{n} \gamma_{i} \delta\left(\mathbf{x}-\mathbf{x}_{i}\right)
$$

with $\delta$ denoting the Dirac measure.

This discretization of the vorticity into a limited number of elements enables to evaluate the velocity field directly from the Bio-Savart integral (equ. 5). Due to the singularity of the Green kernel gradient, $K$, the induced field develops $\frac{1}{r}$-type singularities, where $r$ is the distance to the point vortices.

These singularities can be removed by smoothing the Dirac measure with a cutt-off or blob function, leading to a smoothed version of $K$. Let $f_{\epsilon}$ be such a blob function scaled by a parameter $\epsilon: f_{\epsilon}(\mathbf{x})=\frac{1}{\epsilon^{2}} f\left(\frac{\mathbf{x}}{\epsilon}\right)$. The smoothed kernel is defined as $K_{\epsilon}=$ $K \otimes f_{\epsilon}$. The amount of smoothing is determined by the value of $\epsilon$. If $\epsilon \rightarrow 0, f_{\epsilon}$ tends to the Dirac function and $K_{\epsilon} \rightarrow K$.

In the same way, for the divergence map a source particles representation reads then:

$$
\operatorname{div} \mathbf{w}_{i r r}(\mathbf{x}) \approx \sum_{i=0}^{n} \gamma_{i} f_{\epsilon_{i}}\left(\mathbf{x}-\mathbf{x}_{i}\right)
$$

where $\mathbf{x}_{i}$ denotes the center of each basis function $f_{\epsilon_{i}}$, the coefficient $\gamma_{i}$ is the strength associated to the particle $i$, and $\epsilon_{i}$ represents its influence domain. These parameters are free to vary from a function to another. 


\section{Fluid motion estimation from image sequences}

In this section we present how a vortex and source particles representation may be used in conjunction with an appropriate cost function to devise a motion estimator for image sequences depicting fluid flows.

\subsection{Motion representation}

As we saw previously, discretizing the vorticity map with vortex particles together with a gaussian smoothing of the Dirac measure leads through Bio-Savart integral to the following representation of the solenoidal component of the motion field:

$$
\mathbf{w}_{s o l}(\mathbf{x}) \approx \sum_{i=0}^{n} \gamma_{i}^{s o l} K^{\perp} \otimes f_{\epsilon_{i}}\left(\mathbf{x}-\mathbf{x}_{i}\right)=\sum_{i=0}^{n} \gamma_{i}^{s o l} K_{\epsilon_{i}}^{\perp}\left(\mathbf{x}-\mathbf{x}_{i}\right),
$$

where $K_{\epsilon_{i}}^{\perp}$ is a new kernel function obtained by convolving the orthogonal gradient of the Green kernel with the blob function. Obviously, a similar representation of the irrotational component can be obtained using source particles.

As a result, we exhibit an approximation of the complete motion field as weighted sums of basis functions defined by their centers location and their respective spatial influence. With a gaussian smoothing function which allows to derive analytically the associated smoothed kernel $K_{\epsilon}$, the final expressions of the motion field components are:

$$
\mathbf{w}_{s o l}(\mathbf{x})=\sum_{i=0}^{n^{s o l}} \gamma_{i}^{s o l} \frac{\left(\mathbf{x}-\mathbf{x}_{i}^{s o l}\right)^{\perp}}{2 \pi\left|\mathbf{x}-\mathbf{x}_{i}^{s o l}\right|^{2}}\left(1-e^{-\frac{\left|\mathbf{x}-\mathbf{x}_{i}^{s o l}\right|^{2}}{\epsilon_{i}^{s o l} 2}}\right)
$$

and

$$
\mathbf{w}_{i r r}(\mathbf{x})=\sum_{i=0}^{n^{i r r}} \gamma_{i}^{i r r} \frac{\mathbf{x}-\mathbf{x}_{i}^{i r r}}{2 \pi\left|\mathbf{x}-\mathbf{x}_{i}^{i r r}\right|^{2}}\left(1-e^{-\frac{\left|\mathbf{x}-\mathbf{x}_{i}^{i r r}\right|^{2}}{\epsilon_{i}^{i r r^{2}}}}\right)
$$

This representation will be incorporated within a spatio-temporal variation model of the luminance function in order to devise fluid motion recovery as an estimation problem from the image sequence data.

\subsection{Integrated continuity equation as a brightness variation model}

For image sequences showing evolving fluid phenomena, the usual brightness consistency assumption $\left(\frac{d I}{d t}=0\right)$ doesn't allow to model temporal distortions of luminance patterns caused by 3D matter transportation. For such kind of sequences, several works have shown that a data model build from an analogy with the mass conservation constraint of fluid mechanics (also known as continuity equation) constitutes a better model $[1,4,13,14]$. This data model reads:

$$
\frac{d I}{d t}=I \operatorname{div} \mathbf{w}
$$

Such a constraint relates the effect of a divergent motion to a brightness change. By this way it is possible to modelize the effect of the apparent disappearance/appearance of matter caused by 3D motions which are not in the visualization plane. For a null divergence this data model reduces exactly to the usual brightness consistency equation. 
For long range displacements (i.e. fast flows or long time latency between two images as in meteorology) an integrated form of this constraint can be obtained[4]:

$$
I(\mathbf{x}+\mathbf{w}(\mathbf{x}), t+1) \exp (\operatorname{div} \mathbf{w}(\mathbf{x}))-I(\mathbf{x}, t)=0 .
$$

According to this constraint the displaced image at time $t+1$ is related to the image at time $t$ by a scale factor which depends on the motion divergence. This constraint comes to the standard displaced frame formulation of brightness consistency for a null divergence.

Considering this constraint holds almost everywhere on the whole image plane leads to seek a motion field minimizing the following cost function:

$$
\mathcal{F}(I, \mathbf{w})=\int_{\Omega}[I(\mathbf{x}+\mathbf{w}(\mathbf{x}), t+1) \exp (\operatorname{div} \mathbf{w}(\mathbf{x}))-I(\mathbf{x}, t)]^{2} d \mathbf{x} .
$$

\subsection{General minimization problem}

Considering such a cost function for an unknown motion field approximated through vortex and source particles representations comes down to solve the following minimization problem:

$$
\left(\left\{\hat{\mathbf{x}}_{i}^{\text {sol }}, \hat{\gamma}_{i}^{\text {sol }}, \hat{\epsilon}_{i}^{\text {sol }}\right\}_{i=1: n^{s o l}},\left\{\hat{\mathbf{x}}_{i}^{i r r}, \hat{\gamma}_{i}^{i r r}, \hat{\epsilon}_{i}^{i r r}\right\}_{i=1: n^{i r r}}\right)=\arg \min \mathcal{F}(I, \hat{\mathbf{w}}) .
$$

One seek therefore the minimizer of the cost function $\mathcal{F}$ in terms of particles location, strength coefficients and influence domains. Due to the peculiar form of the data model this minimization problem is highly non linear. To face this difficult optimization problem we have chosen to rely on a non linear least square process embedded in a multi-resolution framework and associated to a generalized conjugated gradient optimization known as Fletcher-Reeves method.

We present more precisely in the next section how this difficult global optimization issue is handled.

\section{Estimation}

The non linear cost function we consider can be seen as a weighted displaced frame $\mathrm{d}$ ifferences cost function. As most of the standard motion estimators based on such a non linear formulation we will consider an incremental minimization framework to remove the non linearity of the displaced image brightness function. This scheme consists in applying successive linearizations around previous estimates. That kind of techniques, in the same spirit as Gauss-Newton non linear least squares, is in most of the case embedded within a multi-resolution framework. We will also rely on such a data representation.

\subsection{Incremental estimation scheme}

We assume first that a previous estimate of the set of unknowns is available. All these unknowns combine together with respect to our modelization to give a motion field $\widetilde{\mathbf{w}}$. Considering a linearization around $(\boldsymbol{x}+\widetilde{\mathbf{w}}, t+1)$ and dropping the time indices of 
the intensity function for sake of clarity we end up with the following functional to be minimized according to $\mathbf{h}$, an unknown correction motion field:

$\mathcal{F}(\mathbf{h})=\int_{\Omega}\left[\exp (\operatorname{div} \tilde{\mathbf{w}}(\mathbf{x}))\left\{(\tilde{I}(\mathbf{x}) \nabla \operatorname{div} \tilde{\mathbf{w}}(\mathbf{x})+\nabla \tilde{I}(\mathbf{x}))^{T} \mathbf{h}(\mathbf{x})+\tilde{I}(\mathbf{x})\right\}-I(\mathbf{x})\right]^{2} d \mathbf{x}$.

In this equation we have introduced a compact notation $\tilde{I}(\mathbf{x})$ for the backward registered image $I(\boldsymbol{x}+\widetilde{\mathbf{w}}, t+1)$. The correction field $\mathbf{h}$ is a combination of a solenoidal component $\mathbf{h}_{\text {sol }}$ and of an irrotational component $\mathbf{h}_{\text {irr }}$ according to the Helmholtz decomposition. Like the field $\widetilde{\mathbf{w}}$, this correction field is parameterized on the basis of a set of vortex and source particles. In practice, this kind of scheme is embedded into a pyramidal multiresolution data representation scheme. Such a representation is obtained through low-pass filtering and sub-sampling. At a given level, the known motion estimate $\tilde{\mathbf{w}}$ is fixed to bev the projected estimate obtained at the previous level. For the first level this field is a null field.

\subsection{Resulting minimization problem}

The incremental estimation scheme transforms the original non linear optimization problem (14) into a succession of simpler minimization problems with respect to some of the unknowns. As a matter of fact, considering the derivatives with respect to the different types of unknowns gives:

$$
\begin{gathered}
\frac{\partial \mathcal{F}(\mathbf{h})}{\partial \gamma_{i}}=\int_{\Omega} \frac{r_{i}(\mathbf{x})}{\pi\left|r_{i}(\mathbf{x})\right|^{2}}\left(1-e^{-\frac{\left|r_{i}(\mathbf{x})\right|^{2}}{\epsilon_{i}{ }^{2}}}\right) y(\mathbf{x})\left[y(\mathbf{x})^{T} \mathbf{h}\left(\mathbf{x}, \gamma_{i}\right)+z(\mathbf{x})\right] d \mathbf{x} \\
\left.\frac{\partial \mathcal{F}(\mathbf{h})}{\partial \beta_{i}}\right|_{\beta_{i}=\frac{1}{\epsilon_{i}}}=\int_{\Omega} \frac{2 \gamma_{i}}{\pi \epsilon_{i}} \frac{r_{i}(\mathbf{x})}{\left|r_{i}(\mathbf{x})\right|^{2}} e^{-\frac{\left|r_{i}(\mathbf{x})\right|^{2}}{\epsilon_{i}{ }^{2}}} y(\mathbf{x})\left[y(\mathbf{x})^{T} \mathbf{h}\left(\mathbf{x}, \epsilon_{i}\right)+z(\mathbf{x})\right] d \mathbf{x} \\
\nabla_{\mathbf{x}_{i}} \mathcal{F}(\mathbf{h})=\left(\begin{array}{c}
\frac{\partial \mathcal{F}(\mathbf{h})}{\partial x_{i}} \\
\frac{\partial \mathcal{F}(\mathbf{h})}{\partial y_{i}}
\end{array}\right)
\end{gathered}
$$

where:

$$
\frac{\partial \mathcal{F}(\mathbf{h})}{\partial x_{i}}=\int_{\Omega}-\frac{\frac{2}{\epsilon_{i}^{2}}\left|r_{i}(\mathbf{x})\right|^{2} r_{i}^{2}(x)+\left(\left|r_{i}(\mathbf{x})\right|^{2}+r_{i}^{2}(x)\right)\left(1-e^{-\frac{\left|r_{i}(\mathbf{x})\right|^{2}}{\epsilon_{i}^{2}}}\right)}{\pi\left|r_{i}(\mathbf{x})\right|^{4}}
$$

and:

$$
\left\{\begin{array}{l}
\beta_{i}=\frac{1}{\epsilon_{i}} \\
r_{i}(\mathbf{x})=\mathbf{x}-\mathbf{x}_{i}=\left(r_{i}(x), r_{i}(y)\right)^{T} \\
y(\mathbf{x})=e^{\operatorname{div} \widetilde{\mathbf{w}}(\mathbf{x})}\left(\tilde{I}_{t+1}(\mathbf{x}) \nabla \operatorname{div} \widetilde{\mathbf{w}}(\mathbf{x})+\nabla \tilde{I}_{t+1}(\mathbf{x})\right) \\
z(\mathbf{x})=e^{\operatorname{div} \widetilde{\mathbf{w}}(\mathbf{x})} \tilde{I}_{t+1}(\mathbf{x})-I_{t}(\mathbf{x})
\end{array}\right.
$$


Canceling out equations (16,17 and 18) leads to three different kinds of systems. The first one, in terms of coefficient strength is linear, the second one in terms of particles influence domain is non linear. No constrained minimization are required for both of them. A gradient descent process can be devised for this set of unknowns. For the third one an additional constraint to keep the particles into the image plane must be added. Such a constrained minimization problem combined with the kind of non linearity we have here leads to a very tough minimization. Besides, if we assume that in some cases we have absolutely no idea of the initial particles location we must devise a method allowing eventual long range moves of the particles coordinates.

We have thus decoupled these three kind of unknowns. The two first (the strength coefficients and the influence domains of the particles) will be solved with a generalized conjugated gradient process while the third kind of unknowns (the particles locations) is kept fixed. The particles location will be in turn updated through a mean shift process that will be described later.

\subsection{Fletcher-Reeves optimization}

Fletcher-Reeves optimization consists in a non linear extension of conjugate gradient algorithms. Given an iterate $\Theta_{k}=\left\{\gamma_{k}^{s o l}, \epsilon_{k}^{s o l}, \gamma_{k}^{i r r}, \epsilon_{k}^{i r r}\right\}$ and a direction $\mathbf{d}_{k}$, a line search (w.r.t. $\alpha_{k}$ ) is performed along $\mathbf{d}_{k}$ to produce $\Theta_{k+1}=\Theta_{k}+\alpha_{k} \mathbf{d}_{k}$. The FletcherReeves variant of the nonlinear conjugate algorithm generates $\mathbf{d}_{k+1}$ from the recursion:

$$
\mathbf{d}_{k+1}=\nabla \mathcal{F}\left(\Theta_{k+1}\right)+\beta_{k} \mathbf{d}_{k} \text { with } \beta_{k}=\left(\frac{\left\|\nabla \mathcal{F}\left(\Theta_{k+1}\right)\right\|_{2}}{\left\|\nabla \mathcal{F}\left(\Theta_{k}\right)\right\|_{2}}\right)^{2}
$$

Let us note that for the linear part of our system the method comes to a standard conjugated gradients. To start the optimization process we consider, as said before that particle locations are fixed. We initialize the domain of influence in an adaptive way. Their values are fixed to the value of the distance to the nearest particles. At convergence, we obtain a representation of the unknown correction field for fixed particle location. Let us now describe how we propose to adjust these locations.

\subsection{Adjustment of particles location}

The estimation method we have proposed requires to fix for the solenoidal and irrotational components particles locations on the image domain. We propose now a way to move each particle according to an error surface defined from the image data. The method we propose is based on the mean shift procedure [8].

Definition of the error function Considering that estimates of the strength coefficients and influence domains are available for both irrotational and solenoidal components we consider two different error surfaces. These surfaces are based first on an image reconstruction criterion. For one given component this criterion measure the discrepancy between the image at time $t$ and the backward registered image $t+1$ obtained considering the other orthogonal component fixed. For the solenoidal component the error surface is defined at each point of the image domain as:

$$
\mathcal{D}^{s o l}(\mathbf{x})=I_{t+1}\left(\mathbf{x}+\tilde{\mathbf{w}}(\mathbf{x})+\tilde{\mathbf{h}}^{i r r}(\mathbf{x})\right)-I_{t}(\mathbf{x}) .
$$


This error surface gather all the reconstruction errors due to the solenoidal component. Similarly the error surface corresponding to the irrotational component is defined as:

$$
\mathcal{D}^{i r r}(\mathbf{x})=I_{t+1}\left(\mathbf{x}+\tilde{\mathbf{w}}(\mathbf{x})+\tilde{\mathbf{h}}^{s o l}(\mathbf{x})\right)-I_{t}(\mathbf{x}) .
$$

The quality of the modelization we consider depends obviously on these error functions but also on the accuracy of the discrete approximation of the divergence and curl map. To achieve the best approximation as possible we should expect to have a great number of particles to describe areas corresponding to divergence or curl blobs and only few of them for the rest of the image. To reach that goal we consider an additional criterion based on the amount of divergence and vorticity. Considering the discrete expressions of the vorticity and of the divergence map given by the particles method we end up with two surfaces:

$$
\mathcal{S}^{s o l}(\mathbf{x})=\frac{\left(\mathcal{D}^{s o l}(\mathbf{x})\right)^{2}}{\sum_{\mathbf{x} \in \Omega}\left(\mathcal{D}^{s o l}(\mathbf{x})\right)^{2}}+\frac{(\operatorname{curl} \tilde{\mathbf{h}}(\mathbf{x}))^{2}}{\sum_{\mathbf{x} \in \Omega}(\operatorname{curl} \tilde{\mathbf{h}}(\mathbf{x}))^{2}},
$$

and

$$
\mathcal{S}^{i r r}(\mathbf{x})=\frac{\left(\mathcal{D}^{i r r}(\mathbf{x})\right)^{2}}{\sum_{\mathbf{x} \in \Omega}\left(\mathcal{D}^{i r r}(\mathbf{x})\right)^{2}}+\frac{(\operatorname{div} \tilde{\mathbf{h}}(\mathbf{x}))^{2}}{\sum_{\mathbf{x} \in \Omega}(\operatorname{div} \tilde{\mathbf{h}}(\mathbf{x}))^{2}},
$$

for the solenoidal and irrotational components respectively. In order to restrict the displacements of the different particles to localized areas we combine these functions with an a priori prior on the particles location.

A priori probability distribution for particles location Considering $\mathbf{x}_{i}^{k}$ the random vector denoting the location of particle $i$ at step $k$, we propose to fix a distribution of $\mathbf{x}_{i}^{m+1}$, knowing $\mathbf{x}_{1: n}^{k}$, where $\mathbf{x}_{1: n}^{k}$ represents the set of the $n$ vectors $\left(\mathbf{x}_{1}^{k}, \ldots, \mathbf{x}_{n}^{k}\right)$ at step $k$. We assume this probability distribution is gaussian, defined as $\mathbf{x}_{i}^{m+1} \mid \mathbf{x}_{1: n}^{m} \sim$ $\mathcal{N}\left(\mathbf{x}_{i}^{m}, \sigma_{i}^{m}\right)$, The standard deviation $\sigma_{i}^{k}$ is set to the half of the distance between $\mathbf{x}_{i}^{m}$ and the closest center among $\left\{\mathbf{x}_{j}^{k}\right\}, j=1, . ., n, j \neq i$. The distribution takes into account the previous location of the particles through a gaussian prior of mean $x_{i}^{k}$ but also the dependency between $\mathbf{x}_{i}^{m+1}$ and all the other particles through the expression of $\sigma_{i}^{m}$.

Conditional version of the probability distribution Combining the a priori distribution $p_{\mathbf{x}_{i}^{m+1} \mid \mathbf{x}_{1: n}^{k}}$ defined above with the surface described before, denoted $\mathcal{S}_{\mathbf{x}_{1: n}^{k}}$ and characterized by (23) or (24), we can define a conditional probability distribution function of a particles $\mathbf{x}_{i}^{k+1}$ given the others:

$$
p_{\mathbf{x}_{i}^{m+1} \mid \mathbf{x}_{1: n}^{m}, \mathcal{S}_{\mathbf{x}_{1: n}^{m}}^{m}}(\mathbf{x}) \propto \mathcal{S}_{\mathbf{x}_{1: n}^{m}}(\mathbf{x}) \cdot p_{\mathbf{x}_{i}^{m+1} \mid \mathbf{x}_{1: n}^{m}}(\mathbf{x}) .
$$

This pdf balances an a priori particle location and the amount of error associated to all the particles locations. Once known this distribution for each particle we propose to shift $\mathbf{x}_{i}^{k}$ towards the pdf local mode in order to find the most probable location of the particles set. 
Shifting the particles towards the pdf modes From the sample $\left\{\mathcal{S}_{\mathbf{x}_{1: n}^{m}}(\mathbf{s})\right\}_{\mathbf{s} \in \mathcal{S}}$ evaluated at pixel coordinates $\mathbf{s}$, and the probability distribution $p_{\mathbf{x}_{i}^{m+1} \mid \mathbf{x}_{1: n}^{m},}$, continuous non parametric estimate of the conditional probability distribution $p_{\mathbf{x}_{i}^{m+1} \mid \mathbf{x}_{1: n}^{m}, \mathcal{S}_{\mathbf{x}_{1: n}^{m}}^{m}}$, may be obtained as

$$
\hat{p}_{\mathbf{x}_{i}^{m+1} \mid \mathbf{x}_{1: n}^{m}, \mathcal{S}_{\mathbf{x}_{1: n}^{m}}}(\mathbf{x}) \propto \frac{\sum_{\mathbf{s} \in \mathcal{S}} \mathcal{S}_{\mathbf{x}_{1: n}^{m}}(\mathbf{s}) p_{\mathbf{x}_{i}^{m+1} \mid \mathbf{x}_{1: n}^{m}}(\mathbf{s}) K\left(\frac{\mathbf{x}-\mathbf{s}}{h}\right)}{\sum_{\mathbf{s} \in \mathcal{S}} K\left(\frac{\mathbf{x}-\mathbf{s}}{h}\right)},
$$

where $K$ is a kernel density and $h$ is the window size.

The continuous pdf $\hat{p}_{\mathbf{x}_{i}^{m+1} \mid \mathbf{x}_{1: n}^{m}, \mathcal{S}_{\mathbf{x}_{1: n}^{m}}^{m}}(\mathbf{x})$ is thus expressed as a linear combination of basis functions with weighted coefficients given by $w(\mathbf{s})=\mathcal{S}_{\mathbf{x}_{1: n}^{m}}(\mathbf{s}) p_{\mathbf{x}_{i}^{m+1} \mid \mathbf{x}_{1: n}^{m}}(\mathbf{s})$.

To shift a center $\mathbf{x}_{i}^{k}$ towards the nearest mode of $\hat{p}_{\mathbf{x}_{i}^{m+1} \mid \mathbf{x}_{1: n}^{m}, \mathcal{S}_{\mathbf{x}_{1: n}^{m}}}$ we rely on the mean shift estimate of the gradient of a density function $[3,8]$. This estimate called the mean shift vector reads:

$$
M_{h, G}(\mathbf{x})=\frac{\sum_{\mathbf{s} \in \mathcal{S}} w(\mathbf{s}) \mathbf{s} G\left(\frac{\mathbf{x}-\mathbf{s}}{h}\right)}{\sum_{\mathbf{s} \in \mathcal{S}} w(\mathbf{s}) G\left(\frac{\mathbf{x}-\mathbf{s}}{h}\right)}-\mathbf{x},
$$

where $G$ is the kernel obtained by derivation of the kernel $K$. This vector gives at each point the direction of the maximum increase of the density function estimated through the weights $w(\mathbf{s})$ and Kernel $K$. Different choices can be done for this kernel. Usual choice are the Epanechnikov kernel or a gaussian kernel. The gradient of the Epanechnikov kernel is a box function kernel whereas $G$ remains gaussian for a gaussian kernel $K$.

Given this estimate of the pdf gradient, an iterative process called mean shift naturally arises, which is convergent [3]. This process consists in moving iteratively the kernel center $z$ :

$$
\mathbf{z}_{k+1}=z_{k}+M_{h, G}\left(\mathbf{z}_{k}\right) .
$$

In our case, the mean shift procedure is applied to the $n_{s o l}+n_{\text {irr }}$ centers of the basis functions (or particles) involved in our motion field modelization. Through this process, each particle is shifted towards the nearest mode of the conditional density $\hat{p}_{\mathbf{x}_{i}^{m+1} \mid \mathbf{x}_{1: n}^{m}, \mathcal{E}_{\mathbf{x}_{1: n}^{m}}^{m}}$. We have chosen to use the Epanechnikov kernel. Besides, the choice of the window size is crucial. Different choices can be made. In our case we have settled adaptative window sizes. They are fixed to the distance of the nearest particles. Such a choice make sense in our case. As a matter of fact, for distant particles only a rough and smooth estimate of the pdf function is needed whereas for close particles an accurate estimate of the density is at the opposite required to approximate at best the vorticity and divergence maps.

\subsection{Overall estimation scheme}

The overall estimation scheme consists in an alternate updating of the different unknowns. It is composed by the following two steps. For a given set of particles at some 
fixed locations, the strength coefficients and the influence domains attached to the particles blob function are estimated through the generalized conjugated gradient optimization described in section 5.3. Once these parameters are updated, the vortex and source particles locations are shifted toward the nearest local mode of the corresponding pdf. This shift is realized applying the mean shift procedure described in the previous section.

The whole process is stopped when the divergence and vorticity reach a certain stability. This criterion is expressed as:

$$
\left(\frac{\left\|\operatorname{div} \mathbf{h}^{n+1}-\operatorname{div} h^{n}\right\|_{2}}{\left\|\operatorname{div} \mathbf{h}^{n}\right\|_{2}}\right)^{2}+\left(\frac{\left\|\operatorname{curl} \mathbf{h}^{n+1}-\operatorname{curl}^{n}\right\|_{2}}{\left\|\operatorname{curl} \mathbf{h}^{n}\right\|_{2}}\right)^{2}
$$

\section{Results}

In this section we present some results given by our method on real sequences.

The first example corresponds to the motion of smoke behind a landing passenger air plane. A strong vortex is located in the center of the image, and a second weaker one begins to appear just below. The particles are initialized on a grid, without a priori. The estimation method allows to guide the vortex particles towards the regions of interest of the image and to estimate an accurate motion field (see the vector field and the associated vorticity map fig. 2. For this sequence we used a multi-resolution pyramid of two levels. At the first level, the particles move all towards the strong vortex (fig. 1 (b) ). At the finest level, the particles cloud splits up into two parts (fig. 1 (c) ). A set of particles has moved towards the weaker vortex, authorizing them to capture its motion.

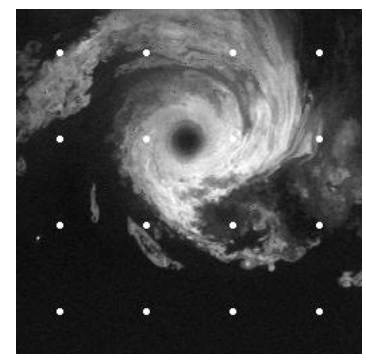

(a)

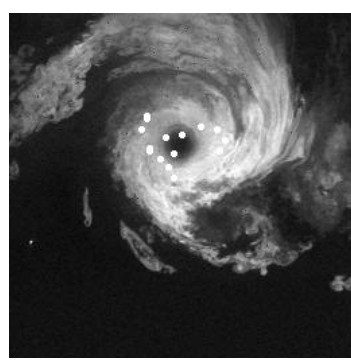

(b)

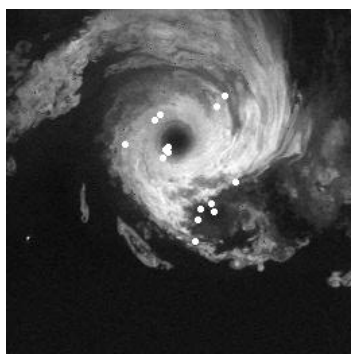

(c)

Fig. 1. Plane sequence. (a) Initial uniform disposition of the particles; (b) Final position of the particles at the first level of multiresolution; (c) Final position of the particles at the second level.

The second example shows results on two consecutive images of the infra-red channel of Meteosat. The sequence represents a depression with a vortex in the left part of the image domain and presence of convective clouds in the center. In this example, we want to observe the motion in specific areas, we dispose thus the vortex and source particles manually in the regions of interest. During the estimation, the particles location fits locally automatically. At convergence, the vortex particles remains mostly concentrated in the center of the vortex, while the source particles are located on the convective cloud. 


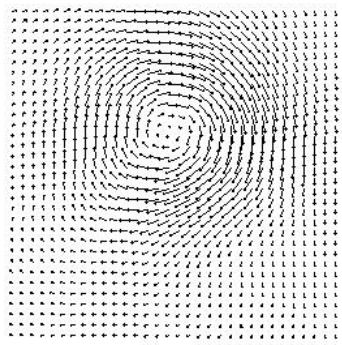

(a)

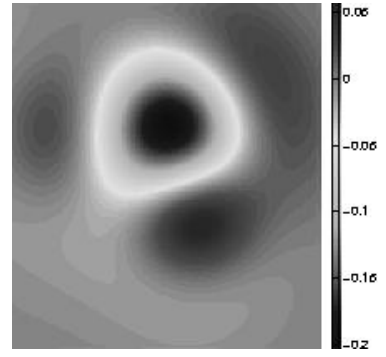

(b)

Fig. 2. Plane sequence. (a) Resulting motion field; (b)Associated vorticity.

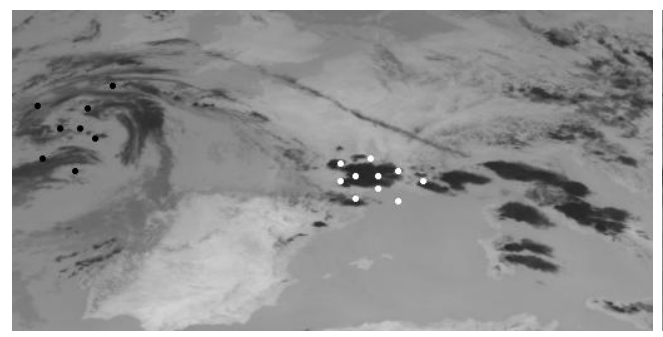

(a)

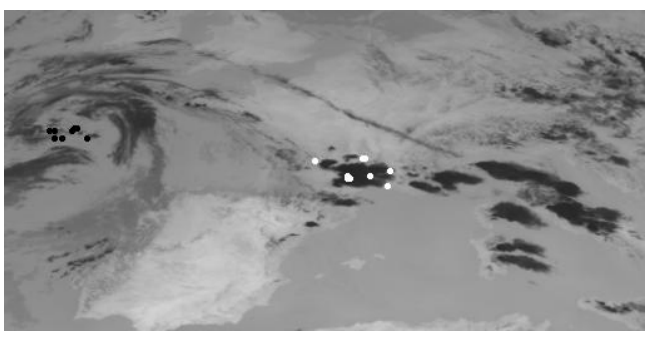

(b)

Fig. 3. Depression sequence. (a) Initial manual disposition of the particles. Black points represent the vortex particles, white points the source ones; (b) Final position of the same particles.

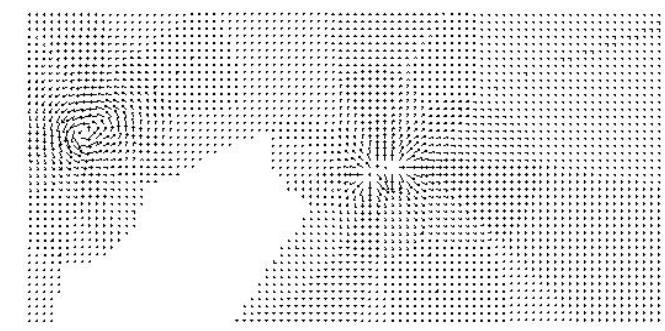

(a)

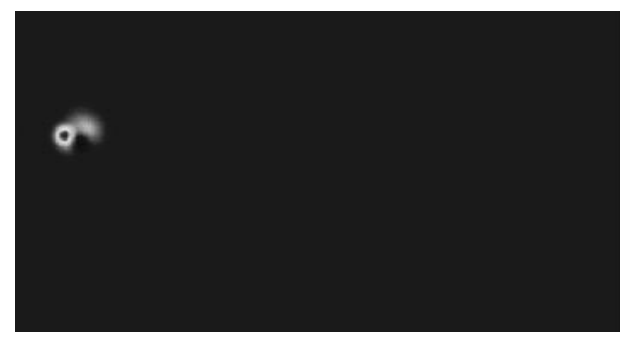

(b)

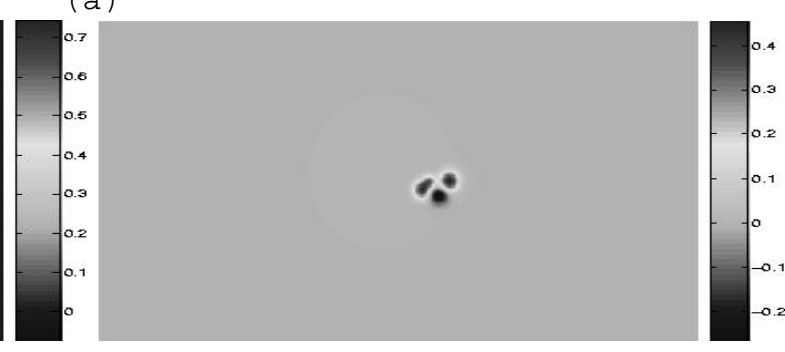

(c)

Fig. 4. Depression sequence. (a) Resulting motion field; (b) Associated vorticity; (c) Associated divergence. 


\section{Conclusion}

In this paper we have presented an optical flow estimator dedicated to image sequences depicting fluid flows. The proposed estimator provides a low dimensional parametric representation of fluid motion. This parameterization has been obtained through a peculiar discretization of the divergence and the vorticity map by means of adapted basis function centered at elements named particles. To handle the associated estimation problem we have proposed an efficient strategy based on the coupling of a generalized conjugated gradient and a mean shift process.

\section{References}

1. D. Béréziat, I. Herlin, and L. Younes. A generalized optical flow constraint and its physical interpretation. In Proc. Conf. Comp. Vision Pattern Rec., volume 2, pages 487-492, Hilton Head Island, South Carolina, USA, 2000.

2. A. Chorin. Numerical study of slightly viscous flow. J. Fluid Mech., 57, 57:785-796, 1973.

3. D. Comaniciu and P. Meer. Mean shift: A robust approach toward feature space analysis. IEEE Trans. Pattern Analysis Machine Intelligence, 24(5):603-619, 2002.

4. T. Corpetti, E. Mémin, and P. Pérez. Dense estimation of fluid flows. IEEE Trans. Pattern Anal. Machine Intell., 24(3):365-380, 2002.

5. T. Corpetti, E. Mémin, and P. Pérez. Extraction of singular points from dense motion fields: an analytic approach. J. Mathematical Imaging and Vision, à paraitre 2003.

6. J.M. Fitzpatrick. A method for calculating velocity in time dependent images based on the continuity equation. In Proc. Conf. Comp. Vision Pattern Rec., pages 78-81, San Francisco, USA, 1985.

7. R.M. Ford, R. Strickland, and B. Thomas. Image models for 2-d flow visualization and compression. Graph. Mod. Image Proc., 56(1):75-93, 1994.

8. K. Fukanaga and L.D. Hostetler. The estimation of the gradient of a density function, with applications in pattern recognition. IEEE Transactions on Information Theory, 21(1):32-40, 1975.

9. T. Kohlberger, E. Mémin, and C. Schnörr. Variational dense motion estimation using the Helmholtz decomposition. In Int. conf on Scale-Space theories in Computer Vision(ScaleSpace '03), Isle of Skye, june 2003.

10. R. Larsen, K. Conradsen, and B.K. Ersboll. Estimation of dense image flow fields in fluids. IEEE trans. on Geoscience and Remote sensing, 36(1):256-264, Jan. 1998.

11. A. Leonard. Vortex methods for flow simulation. J. Comp. Phys., 37, 1980.

12. E. Mémin and P. Pérez. Fluid motion recovery by coupling dense and parametric motion fields. In Int. Conf. on Computer, ICCV'99, pages 620-625, 1999.

13. B.G. Schunk. The motion constraint equation for optical flow. In Proc. Int. Conf. Pattern Recognition, pages 20-22, Montreal, 1984.

14. R. Wildes, M. Amabile, A.M. Lanzillotto, and T.S. Leu. Physically based fluid flow recovery from image sequences. In Proc. Conf. Comp. Vision Pattern Rec., pages 969-975, 1997.

15. L. Zhou, C. Kambhamettu, and D. Goldgof. Fluid structure and motion analysis from multispectrum 2D cloud images sequences. In Proc. Conf. Comp. Vision Pattern Rec., volume 2, pages 744-751, Hilton Head Island, South Carolina, USA, 2000. 